\title{
Effect of Baseline RDW and Troponin Levels on Prognosis in Patients with Acute Chest Pain
}

\author{
Hatice Şeyma Akça ${ }^{1, \star}$, Abdullah Algın ${ }^{1}$, Serdar Özdemir ${ }^{1}$, İbrahim Altunok ${ }^{1}$, \\ Sümeyra Acar Kurtuluş ${ }^{1}$, Serkan Emre Eroğlu ${ }^{1}$
}

${ }^{1}$ Department of Emergency Medicine, University of Health Sciences, Ümraniye Education and Research Hospital, Istanbul, Turkey

\section{*Correspondence}

drhaticeseyma_@hotmail.com

(Hatice Şeyma Akça)

\begin{abstract}
Introduction: Red cell distribution width (RDW) is a measure of the mean corpuscular/erythrocyte volume. The aim of this study was to investigate the effect of RDW levels on the prognosis in patients with elevated levels of troponin I in the emergency department with chest pain. Materials and methods: The study included 3,922 patients aged over 18 years who presented to the emergency department with the complaint of chest pain between August 1, 2018 and August 1, 2019. Troponin values and RDW levels were compared to determine the ability to diagnose acute ischemic heart disease and determin the prognosis in these patients. Results: The median RDW was 13.3 (IQR 1.20) in patients with normal troponin and 13.3 (IQR 1.40) in patients with elevated troponin $(\mathrm{p}=0.001)$, but there was no difference in clinical outcomes between the groups. There was no significant difference in RDW between the patients discharged from the emergency department, those discharged from the hospital (good outcome) and those that required intensive care or died (poor outcome) $(p>0.05$ ). Conclusion: RDW levels may vary in patients with elevated troponin values and acute ischemia, but this has no affect on clinical outcomes.
\end{abstract}

\section{Keywords}

Chest pain, Myocardial infarction, RDW

\section{Introduction}

Red cell distribution width (RDW) is a measure of the mean corpuscular/erythrocyte volume calculated using the standard deviation of erythrocyte volume heterogeneity. Endothelial dysfunction, hypoxia, inflammation and ischemic clinical events are associated with Red cell distribution width (RDW) [1]. Elevated Red cell distribution width (RDW) is associated with anemia, especially the microcytic type. It is also used in the differential diagnosis of Fe deficiency anemia and heterozygous thalassemia. The Red cell distribution width (RDW) may increase in B12 deficiency, folate deficiency, and hemolytic anemia [2]. Advanced age, genetic factors, lifestyle, and comorbidities increase the risk of developing cardiovascular disease and its associated morbidity and mortality. It has been suggested that cardiovascular diseases may be related to hemogram parameters in addition to known risk factors, such as lipid profile, atherogenic index, and cholesterol level, and various inflammatory parameters. It has also been suggested that diagnosis and treatment decisions can be made based on markers, such as the mean platelet volume, mean erythrocyte volume, and total bilirubin. Similarly, RDW levels are among risk factors for cardiovascular disease [3-7]. Although the mechanism of elevated RDW on cardiovascular system diseases and complications is not yet fully known, it has been stated that a decrease in oxygen capacity may cause ischemia. RDW has been found to be a risk factor for mortality independent of mean corpuscular volume (MCV) and other hematological factors $[8,9]$. The aim of this study was to investigate the effect of RDW levels in patients with elevated troponin I levels who presented to the emergency department with chest pain; and its affect on prognosis.

\section{Materials and methods}

\subsection{Patient population}

This study included patients over 18 years of age who presented to the emergency department with the complaint of chest pain between August 1, 2018 and August 1, 2019. The Emergency Department of Ümraniye Training and Research Hospital is a comprehensive clinic serving an average of 500,000 patients every year with green, yellow and red zones and a resuscitation unit. Patients with available data on the troponin-I and Red cell distribution width (RDW) values at the time of presentation were included in this retrospective study while those with incomplete laboratory findings, unavailable electrocardiography (ECG) findings, chest pain due to trauma, and the diagnosis of psychiatric diseases were excluded. The patients' initial troponin I and RDW values were compared to determine prognosis. Hospital data system records were used to determin patients discharged from the emergency 


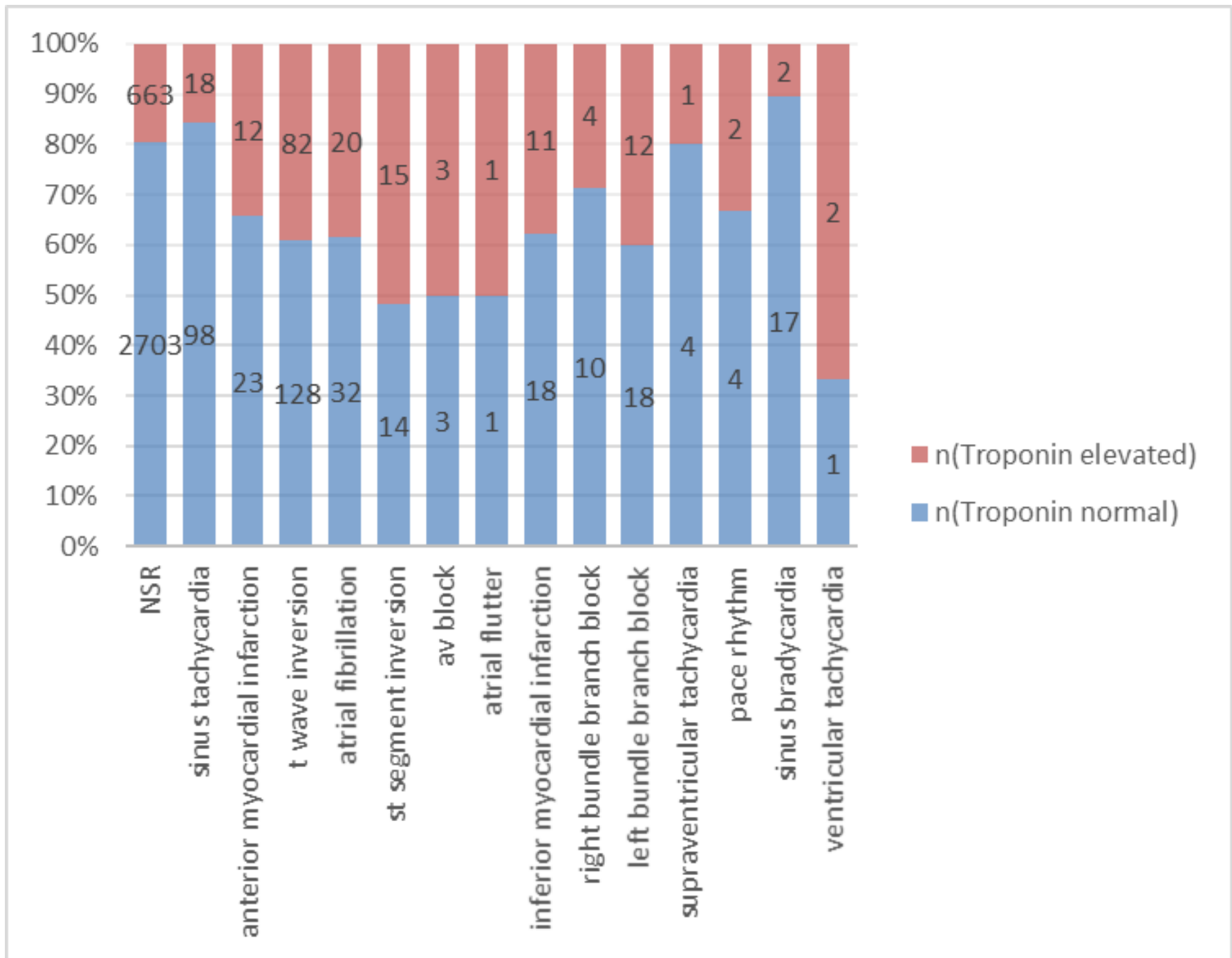

F I G U R E 1. ECG findings of patients with normal and high troponin I values.

department, those discharged from the hospital and those who died during hospitalization. The demographic data, medical history, electrocardiography (ECG) findings, Red cell distribution width (RDW), mean platelet volume (MPV), initial troponin I values, and discharge rates were recorded.

The patients were divided into three groups according to clinical outcome: those discharged from the emergency department without being hospitalized, those with a good prognosis who were discharged from a hospital service, and those with a poor prognosis who required intensive care or died.

\subsection{Laboratory Measurements}

Complete blood count and RDW analysis were performed with Cell-Dynn 3700 (Abott, IL, USA). Cardiac troponin measurements were made with ARCHITECH ci400 (ARCHITECH, IL, USA) by the indirect ion-selective electrode dilution method.

The normal cut off value of troponin I was $<0.0262 \mathrm{ng} / \mathrm{mL}$, patients with a troponin I value less than $0.02 \mathrm{ng} / \mathrm{mL}$ were included in the normal troponin I group while those with a value higher than $0.02 \mathrm{ng} / \mathrm{mL}$ were evaluated as having a high troponin I. The normal range of RDW levels was $11-16 \%$. Patients with RDW > 16\% were termed high RDW levels; patients with RDW $<11 \%$ were termed low RDW levels. The normal range of MPV levels was 6 - $12 \mathrm{fL}$.

\subsection{Statistical analyses}

Statistical analysis was performed using SPSS version 22. The conformance of variables to normal distribution was examined by visual (histogram and probability graphs) and analytical methods (Kolmogorov-Smirnov/Shapiro-Wilk test). Descriptive analyses were given for the median and interquartile range (frequency tables for ordinal variables) for non-normally distributed data. Since it was determined that the blood parameters with numerical data were not normally distributed, these parameters and ordinal data; i.e., clinical outcome and ECG variables were compared between the groups using the Kruskal-Wallis test. The Mann- Whitney U test was performed to test the significance of pairwise differences using Bonferroni correction to adjust for multiple comparisons. While investigating the associations between non-normally distributed and/or ordinal variables, the correlation coefficients and their significance were calculated using the spearman test. A p value of less than 0.05 was considered statistically significant. 


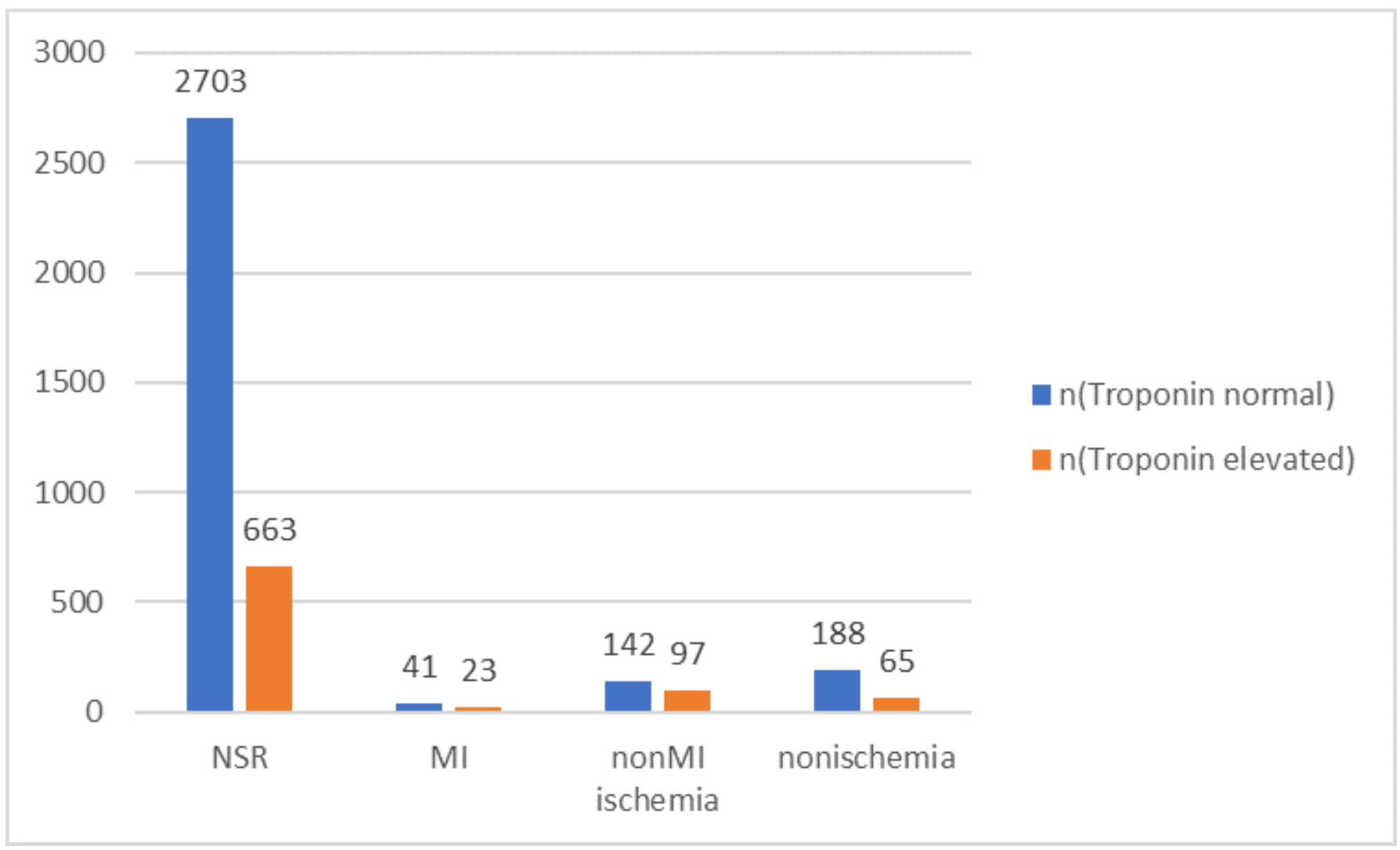

F I G U R E 2. Evaluation of the ECG findings of normal sinus rhythm (NSR), myocardial infarction (MI), ischemia, nonischemia, and according to troponin I values.

\section{Results}

The study included 3,922 patients. Of the 3,922 patients, $2,196(56 \%)$ were male and $1,726(44 \%)$ were female. The median age of the patients discharged from the emergency room was 46 [25], the median age of the patients with better prognosis was 46 [25], and the median age of poor prognosis was 38 [13]. 392 patients were hospitalized and progressed with better prognosis. 7 patients had poor prognosis, and 3522 patients were discharged after completion of the emergency examination and treatment procedures. The presence of comorbid diseases was higher in patients with poor prognosis. Discharge from the hospital resulted in a better prognosis $(\mathrm{p}<$ 0.05 ). When biochemical values, hemogram parameters and vital signs were evaluated according to prognosis, there was no statistically significant difference between the three groups (p > 0.05) (Table 1).

The most common ECG rhythm was normal sinus rhythm (NSR) observed in 3,366 (85\%) patients. The relationship between the remaining ECG findings and high troponin I was recorded in Fig. 1. 14.3\% of MI findings were detected in the first-visit ECG of the patients resulting in better prognosis. Ischemic changes without MI findings were detected in $28.6 \%$ of patients resulting in poor prognosis. There was a significant difference between the ECG parameters of the patients and prognosis $(\mathrm{p}<0.00)$ (Table 1$)$.

According to the ECG parameters, the patients were divided into four groups; those with normal sinus rhythm (NSR), those with findings consistent with acute myocardial infarction (MI), those with ischemic findings other than MI, and those with no ischemic finding but without normal sinus rhythm (NSR) (Fig. 2). While $87.9 \%$ of the patients with normal troponin I had NSR, $78.2 \%$ of those with high troponin I had normal sinus rhythm (NSR), indicating no clinically significant difference between the groups $(\mathrm{p}>0.001)$ (Fig. 2). However, there was a statistically significant difference between the patients with high and normal troponin I in terms of the ischemic ECG findings other than MI $(\mathrm{p}<0.001)$.

There was no statistically significant difference in the RDW values, categorized RDW and troponin levels according to gender $(\mathrm{p}=0.837 ; \mathrm{p}=0.277 ; \mathrm{p}=0.816$; respectively). The relationships of clinical outcome with high and normal troponin I, and categorized Red cell distribution width (RDW) in patients are shown in Table 2. According to Troponin I variables, there was a statistically significant difference in terms of clinical outcome, and according to the Games-Howell posthoc analysis, the difference was seen between better clinical outcome and emergency discharge $(p<0.001)$. Another hemogram parameter, the MPV (mean platelet volume) level was found to have a median value of $9.5 \mathrm{fL}$ (IQR 1.20) in the group with normal troponin I and $9.5 \mathrm{fL}$ (IQR 1.20) in the group with high troponin I, indicating no statistically significant difference $(\mathrm{p}>0.05)$. When the RDW levels were compared in terms of clinical outcome, there was no statistically significant difference between the patients discharged from the emergency department without hospitalization, those discharged from a hospital service (better prognosis), and those requiring intensive care or died (poor prognosis) $(\mathrm{p}>0.05)$ (Spearman's correlation analysis: $\mathrm{r}=-0.025, \mathrm{p}=0.110$ ). 
TA B L E 1. Demographic data, laboratory findings, known diseases of the patients.

\begin{tabular}{|c|c|c|c|c|}
\hline & Discharge & Better prognosis & Poor prognosis & $\mathrm{p}$ \\
\hline Age & $46(25)$ & $46(25)$ & $38(13)$ & $>0.05$ \\
\hline Gender(n) & 3523 & 392 & 7 & \\
\hline Male(\%) & $55.7 \%$ & $58.4 \%$ & $71.4 \%$ & \\
\hline Female $(\%)$ & $44.3 \%$ & $41.6 \%$ & $28.6 \%$ & $>0.05$ \\
\hline \multicolumn{5}{|l|}{ Vital signs } \\
\hline SBP(mmhg) & $125(60-152)$ & $128.5(80-220)$ & $129(118-160)$ & $>0.05$ \\
\hline $\mathrm{DBP}(\mathrm{mmhg})$ & $80(70-89)$ & $78(65-92)$ & $82(46-100)$ & $>0.05$ \\
\hline $\mathrm{HR}(/ \min )$ & $82(45-200)$ & $81(41-170)$ & $88(65-97)$ & $>0.05$ \\
\hline $\mathrm{sPO} 2(\%)$ & $98(64-100)$ & $98(87-100)$ & $97(95-98)$ & $>0.05$ \\
\hline ECG categories $(\%)$ & & & & $<0.001$ \\
\hline NSR & 87 & 75.3 & 71.4 & \\
\hline MI & 0.2 & 14.3 & 0 & \\
\hline nonMI ischemic changes & 6.2 & 4.8 & 28.6 & \\
\hline non-ischemic changes & 6.6 & 5.6 & 0 & \\
\hline \multicolumn{5}{|l|}{ Comorbidities\% (+/-) } \\
\hline HT & $14 / 86$ & $22.4 / 77.6$ & $28.6 / 71.4$ & $<0.05$ \\
\hline $\mathrm{DM}$ & $8.8 / 91.2$ & $12.5 / 87.5$ & $14.3 / 85.7$ & $=0.049$ \\
\hline CAD & $6.6 / 93.4$ & $10.7 / 89.3$ & $28.6 / 71.4$ & $<0.05$ \\
\hline $\mathrm{CRF}$ & $0.7 / 99.3$ & $1.3 / 98.7$ & $28.6 / 71.4$ & $<0.05$ \\
\hline HPL & $3.7 / 96.3$ & $7.1 / 92.9$ & $14.3 / 85.7$ & $<0.05$ \\
\hline \multicolumn{5}{|l|}{ BK parameters } \\
\hline Glucose(mg/dL) & $103(50-610)$ & $105(70-470)$ & $99(89-134)$ & $\mathrm{p}>0.05$ \\
\hline BUN (mg/dL) & $29(20-49)$ & $29.9(20-117)$ & $34.2(23.5-81.3)$ & $\mathrm{p}>0.05$ \\
\hline Cre (mg/dL) & $0.8(0.5-9)$ & $0.8(0.3-6)$ & $0.8(0.6-8.3)$ & $\mathrm{p}>0.05$ \\
\hline $\mathrm{Na}(\mathrm{mEq} / \mathrm{L})$ & $139(100-149)$ & $139(98-146)$ & $137(123-140)$ & $\mathrm{p}>0.05$ \\
\hline $\mathrm{K}(\mathrm{mEq} / \mathrm{L})$ & $4.3(2.8-5.9)$ & $4.3(3.2-5.7)$ & $4.5(3.6-4.9)$ & $\mathrm{p}>0.05$ \\
\hline \multicolumn{5}{|l|}{ Hemogram parameters } \\
\hline WBC $\left(10^{3} \mu \mathrm{L}\right)$ & $8.5(5-29.5)$ & $8.8(1.17-18)$ & $7.3(6.4-15.1)$ & $p>0.05$ \\
\hline Neu $\left(10^{3} \mu \mathrm{L}\right)$ & $5(2-26.7)$ & $5.1(0.7-15)$ & $5.9(3.4-11)$ & $\mathrm{p}>0.05$ \\
\hline $\operatorname{Hgb}(\mathrm{g} / \mathrm{dl})$ & $13.8(11-19.5)$ & $13.8(6.1-17.9)$ & $12.3(10.1-15.4)$ & $\mathrm{p}>0.05$ \\
\hline $\operatorname{Hct}(\%)$ & $41.3(35-55)$ & $41.6(19.3-54.1)$ & $36.2(30.7-46.4)$ & $\mathrm{p}>0.05$ \\
\hline $\operatorname{RDW}\left(10^{3 /} \mu \mathrm{L}\right)$ & $13.3(8.1-26.5)$ & $13.4(11.6-23.7)$ & $13.9(12.7-18.6)$ & $\mathrm{p}>0.05$ \\
\hline $\operatorname{MCV}(\mathrm{fL})$ & $86.7(75-112.5)$ & $87.2(63.9-99.5)$ & $86.4(78.9-92)$ & $\mathrm{p}>0.05$ \\
\hline Plt $\left(10^{3} \mu \mathrm{L}\right)$ & $251.6(145-830)$ & $251(111-508)$ & $215(171-273)$ & $\mathrm{p}>0.05$ \\
\hline $\operatorname{MPV}\left(10^{3} \mu \mathrm{L}\right)$ & $9.5(7-14.5)$ & $9.5(6.1-12.6)$ & $10.1(5.8-10.7)$ & $\mathrm{p}>0.05$ \\
\hline
\end{tabular}

SBP: systolic blood pressure, DBP: diastolic blood pressure, HR: heart rate, NSR: normal sinus rhythm, MI: myocardial infarction, HT: hypertension, DM: diabetes mellitus, CRF: Chronic renal failure, HPL: hyperlipidemia, BUN: blood urea nitrogen, Crea: creatinine, Na: sodium, K: potassium, WBC: white blood cell, Neu: neutrophil, Hgb: hemoglobine, Hct: hematocrit, RDW: red cell distribution width, MCV: mean corpuscular volume, Plt: platelet, MPV: mean platelet volume.

When RDW and troponin levels variable were compared, there was a statistically significant relationship between them and there was very weak positive correlation. $0.3 \%$ of the change in troponin level varied with RDW $(p=0.001)$ (Spearman's correlation analysis: $\mathrm{r}=0.055, \mathrm{p}=0.3$ ).

Patients with elevated troponin were consulted with cardi- ology and no emergency cardiac pathology were discharged after follow-up and treatment in the emergency room. HT in $256(35.9 \%)$ of these 714 patients, hypoxia in $244(34.2 \%)$, hypoperfusion in $120(16.8 \%)$, tachyarrhythmia in $52(7.3 \%)$, anemia in $33(4.6 \%)$, bradyarrhythmia in $8(1.1 \%)$, and chronic renal failure in $1(0.1 \%)$. 
TA B L E 2. Evaluation of RDW and troponin I values with prognosis.

\begin{tabular}{|c|c|c|c|c|c|}
\hline & & Discharge & Better prognosis & Poor prognosis & $\mathrm{p}$ \\
\hline \multirow[t]{3}{*}{ Troponin I (ng/ml) } & & & & & $<0.001$ \\
\hline & Normal troponin I & $2809(79.7 \%)$ & $263(67.1 \%)$ & $2(28.6 \%)$ & \\
\hline & Elevated troponin I & $714(20.3 \%)$ & $129(32.9 \%))$ & $5(71.4 \%)$ & \\
\hline \multirow[t]{4}{*}{$\operatorname{RDW}\left(10^{3} / \mu \mathrm{L}\right)$} & & & & & $>0.05$ \\
\hline & $<11 \%$ & $8(0.2 \%)$ & & & \\
\hline & $11-16 \%$ & $3264(92.7 \%)$ & $371(94.6 \%)$ & $6(85.7 \%)$ & \\
\hline & $>16 \%$ & $250(7.1 \%)$ & $21(5.4 \%)$ & $1(14.3 \%)$ & \\
\hline
\end{tabular}

$R D W$, red cell distribution width.

TA B L E 3. Distribution of ECG changes according to prognosis in patients with troponin I $>0.002 \mathrm{ng} / \mathrm{ml}$.

\begin{tabular}{lccc} 
& Discharge & Better prognosis & Poor prognosis \\
NSR & $564(79 \%)$ & $96(74.4)$ & $3(60 \%)$ \\
MI & $6(0.8 \%)$ & $17(13.2 \%)$ & \\
Non MI ischemia & $85(11.9 \%)$ & $10(7.8 \%)$ & $2(40 \%)$ \\
Non ischemia & $59(8.3 \%)$ & $6(4.7 \%)$ & \\
Total & 714 & 129 & 5 \\
\hline
\end{tabular}

When the ECG findings and prognosis of patients with elevated troponin were examined, NSR was detected in $79 \%$ of patients discharged, $74.4 \%$ of patients with better prognosis and $60 \%$ of patients with poor prognosis. $13.2 \%$ of the patients with better prognosis and $0.8 \%$ of the patients discharged from the emergency department had an MI finding on the first-time ECG (Table 3).

\section{Discussion}

In this study, when the RDW levels were compared in terms of clinical outcome, there was no statistically significant difference between the patients discharged from the emergency department without hospitalization, those discharged from a hospital service (better prognosis), and those requiring intensive care or died (poor prognosis) $(\mathrm{p}>0.05)$ (Spearman's correlation analysis: $r=-0.025, p=0.110)$. When RDW and troponin levels variable were compared, there was a statistically significant relationship between them and there was very weak positive correlation. $0.3 \%$ of the change in troponin level varied with RDW ( $\mathrm{p}=0.001)$.

Although the gold standard for the diagnosis of an acute MI is a high troponin I value, changes in MPV and RDW levels have also been studied to determine their ability to facilitate diagnosis. There was no poor prognosis in our patients with MI findings on the first-arrival ECG. Undoubtedly, these changes resulted in earlier interventions. Early intervention in ischemic ECG changes was very important. The most common ECG changes in patients with poor prognosis were those without MI findings but with ischemic changes on ECG. In unstable angina, acute $\mathrm{MI}$ and sudden cardiac death, ischemia triggers platelet activation which results in elevated MPV. In a study in which patients with non-ST elevation were followed up for three years, aspirin resistance and elevated MPV were found to increase the risk of developing a reccurent MI [6]. In another study, in which patients with non-ST elevation were examined, it was found that high RDW was an independent determinant of one-year mortality in patients that were hospitalized for the first time due to heart failure [8].

In our study, with the increase in RDW levels, discharge was reduced. Patients with high RDW were discharged after hospitalization or intensive care hospitalization with poor prognosis. Many studies have been conducted to evaluate whether hemogram parameters can be prognostic factors. Similarly, it has been investigated whether there is any relation between various parameters causing hypoxia and ischemia and the troponin I level. In a study that aimed to determine the possible association between the ionized calcium level and mortality in cases of pulmonary embolism, a low correlation was observed between the troponin I level and ionized calcium[10]. There are many reasons for increases in the Troponin I level. In our study, we detected hypertensive crisis, hypoxia and hypoperfusion in patients who had high troponin I level and were discharged from the emergency department. In a study, normal RDW values were found in $20 \%$ of cases with FE deficiency anemia, and RDW was elevated in half of those with chronic disease anemia and heterozygous thalassemia [11]. Other researchers reported that increased neutrophil/lymphocyte ratio was associated with increased mortality in patients receiving intensive care [12]. Similarly, studies in the literature showed that MPV was associated with intravascular thrombus and altered platelet functions $[13,14]$. All these findings suggest that the variability of RDW due to hypoxia and ischemia may determine diagnosis and prognosis. In our study there was no correlation between the discharge status of patients and their RDW values. Although a statistically significant difference was found between the troponin I and RDW levels $(p=0.001)$, there was no statistically significant difference between better prognosis, poor prognosis, and RDW levels $(p>0.05)$. This suggests that it may not be appropriate to use RDW values to 
determine prognosis in these patients.

In some studies, average RDW values have been reported to be higher in women $[11,15,16]$ while others found no significant relationship between gender and RDW values [11, 17-20]. In our study, there was no statistically significant difference in the RDW values, categorized RDW and troponin levels according to gender $(\mathrm{p}=0.837 ; \mathrm{p}=0.277 ; \mathrm{p}=0.816$; respectively). There are also studies revealing that the RDW value is affected by obesity and metabolic syndromes, and exercise can result in a significant improvement in cardiovascular disease and RDW [11,21, 22]. Although it is suggested that RDW may be affected by genetic factors, it should be remembered that hyperlipidemia, hypertension, and cardiovascular disease may also be genetic, and RDW may increase due to these diseases [11]. Comorbid diseases were distributed according to prognosis and the presence of comorbid disease was higher in patients with poor prognosis. There was a significant difference in all groups discharged, resulting in better prognosis and poor prognosis $(\mathrm{p}<0.05)$.

In the literature, studies have also evaluated the relationship between RDW and coronary artery diseases in patients that have undergone angioplasty [5, 23, 24]. In patients with MI that have undergone this procedure, a relationship has been reported between the RDW level and mortality $[5,25,26]$. In our study, our patients with a better prognosis were those who had been hospitalized and underwent angioplasty. There was no significant relationship between better prognosis and RDW values. Although the underlying reason for the relationship between RDW and cardiovascular diseases is not yet precisely known, it may be associated with proinflammatory cytokines and oxidative stress increasing the RDW level [5, 27, 28]. Despite the statistically significant difference in the RDW levels between our normal and high troponin I groups, no clinical significance was observed in this evaluation.

\section{Conclusion}

Although it can be shown that RDW, one of the hemogram parameters, can be effective in determining the prognosis of patients with ischemic pathology; detailed meta-analysis is needed.

RDW levels may vary in patients with elevated troponin levels and chest pain but they have no effect on clinical outcomes.

\section{LIMITATIONS}

Since our study was retrospective only patients with 'chest pain' were examined. Patients diagnosed with acute coronary syndrome with different complaints could not be evaluated.

Our patients were only given a blood test at the time of presentation with no follow-up analysis being conducted; thus, antiplatelet use was not determined. We may not have been able to distinguish patients who had not yet been diagnosed with hypertension, diabetes mellitus, or cardiac disease but carried the associated risk factors. In addition, the affects of revascularization procedures such as angioplasty was not determined. Lastly, readmissions were not evaluated.

\section{ACKNOWLEDGMENTS}

Thanks to all the peer reviewers and editors for their opinions and suggestions.

\section{CONFLICT OF INTEREST}

The authors declare no conflict of interest.

\section{ETHICAL APPROVAL}

Our study was approved on 24.09.2019 with the decision of the Clinical Research Ethics Committee of Ümraniye Training and Research Hospital.

\section{REFERENCES}

[1] Dursun M, Cimen S, Sulukaya M, et al. The predictive value of red cell distribution width on erectile dysfunction. Andrologia. 2019;5:13374.

[2] Fava C, Cattazzo F, Hu Z, et al. The role of red blood cell distribution width (RDW) in cardiovascular risk assessment: useful or hype? Ann Transl Med. 2019;7:581.

[3] Parizadeh SM, Jafarzadeh-Esfehani R, Bahreyni A, et al. The diagnostic and prognostic value of red cell distribution width in cardiovascular disease; current status and prospective. BioFactors, 2019;4:507-516.

[4] Payne RA. Cardiovascular risk. Br J Clin Pharmacol. 2012;74: 396-410.

[5] Wu T, Zheng Y, Hou X, et al. Red blood cell distribution width as long-term prognostic markers in patients with coronary artery disease undergoing percutaneous coronary intervention. Lipids Health Dis. 2019;18:140.

[6] Aksu H, Ozer O, Unal H, et al. Significance of mean platelet volume on prognosis of patients with and without aspirin resistance in settings of non-ST-segment elevated acute coronary syndromes. Blood Coagul Fibrinolysis. 2009;20:686-693.

[7] Troughton JA, Woodside JV, Young IS, et al. PRIME Study Group. Bilirubin and coronary heart disease risk in the prospective epidemiological study of myocardial infarction (PRIME). Eur J Cardiovasc PrevRehabil. 2007;14:79-84.

[8] Salvatori M, Formiga F, Moreno-Gonzales R, et al. Red blood cell distribution width as a prognostic factor of mortality in elderly patients firstly hospitalized due to heart failure. Kardiol Pol. 2019;6:632-638.

[9] Wołowiec L, Rogowicz D, Banach J, et al. Prognostic significance of red cell distribution width and other red cell parameters in patients with chronic heart failure during two years of follow-up. Kardiol Pol. 2016;74:657-664.

[10] Akça HŞ, Koylu R, Akıllı NB, et al. Effect of Ionized Calcium Level on the Prognosis and Mortality of Patients with Pulmonary Embolism. American Journal of Internal Medicine. 2018;1:20-24.

[11] Poz D, De Falco E, Pisano C, et al. Diagnostic and prognostic relevance of Red blood cell distribution width for vascular aging and cardiovascular diseases. Rejuvenation Research. 2018;22:146-162.

[12] Akilli NB, Yortanlı M, Mutlu H, et al. Prognostic importance of neutrophil-lymphocyte ratio in critically ill patients: short- and long-term outcomes, Am J Emerg Med. 2014;32:1476-1480.

[13] Monteiro Junior J G de M, De Oliveira Cipriano Torres D, Filho D C $\mathrm{S}$. Hematological parameters as prognostic biomarkers in patients with cardiovascular diseases. Curr Cardiol Rev. 2019;15:274-282.

[14] Wendland AE, Farias MG, Manfroi WC. Volume plaquetáriomédio e doença cardiovascular. J BrasileiroPatologiamedicina Laboratorial. 2009;45:371-378.

[15] Lippi G, Salvagno GL, Guidi GC. Red blood cell distribution width is significantly associated with aging and gender. Clin Chem Lab Med. 2014;52:197-199.

[16] Alis R, Fuster O, Rivera L, et al. Influence of age and gender on red blood cell distribution width. Clin Chem Lab Med. 2015;53:25-28.

[17] Abrahan LL, Ramos JDA, Cunanan EL, et al. Red Cell Distribution Width 
and Mortality in Patients With Acute Coronary Syndrome: A MetaAnalysis on Prognosis. Cardiol Res. 2018;9:144-152.

[18] Cheng CK, Chan J, Cembrowski GS, et al. Complete blood count reference interval diagrams derived from NHANES III: Stratification by age, sex, and race. Lab Hematol. 2004;10:42-53.

[19] Chen PC, Sung FC, Chien KL, et al. Red blood cell distribution width and risk of cardiovascular events and mortality in a community cohort in Taiwan. Am J Epidemiol. 2010;171:214-220.

[20] Qiao R, Yang S, Yao B, et al. Complete blood count reference intervals and age- and sex-related trends of North China Han population. Clin Chem Lab Med. 2014;52:1025-1032.

[21] Furuncuoglu Y, Tulgar S, Dogan AN, et al. How obesity affects the neutrophil/lymphocyte and platelet/lymphocyte ratio, systemic immuneinflammatory index and platelet indices: A retrospective study. Eur Rev Med Pharmacol Sci. 2016;20:1300-1306.

[22] Loprinzi PD, Hall ME. Physical activity and dietary behavior with red blood cell distribution width. PhysiolBehav. 2015;149:35-38.

[23] Ye Z, Smith C, Kullo IJ. Usefulness of red cell distribution width to predict mortality in patients with peripheral artery disease. Am J Cardiol. 2011;107:1241-1245.

[24] Osadnik T, Strzelczyk J, Hawranek M, et al. Red cell distribution width is associated with longterm prognosis in patients with stable coronary artery disease. BMC Cardiovasc Disord. 2013;13:113.

[25] Uyarel H, Ergelen M, Cicek G, et al. Red cell distribution width as a novel prognostic marker in patients undergoing primary angioplasty for acute myocardial infarction. Coron Artery Dis. 2011;22:138-144.

[26] Fatemi O, Paranilam J, Rainow A, et al. Red cell distribution width is a predictor of mortality in patients undergoing percutaneous coronary intervention. J Thromb Thrombolysis. 2013;35:57-64.

[27] Rechavi G, Rivella S. Regulation of iron absorption in hemoglobinopathies. Curr Mol Med. 2008;8:646-662.

[28] Schieffer B, Schieffer E, Hilfiker-Kleiner D, et al. Expression of angiotensin II and interleukin 6 in human coronary atherosclerotic plaques:potential implications for inflammation and plaque instability. Circulation. 2000;101:1372-1378.

How to cite this article:Hatice Şeyma Akça, Abdullah Algın, Serdar Özdemir, İbrahim Altunok, Sümeyra Acar Kurtuluş, Serkan Emre Eroğlu. Effect of Baseline RDW and Troponin Levels on Prognosis in Patients with Acute Chest Pain. Signa Vitae. 2020;16(2):97-103. doi:10.22514/sv.2020.16.0050. 